\title{
Design study for the cascaded HGHG experiment based on the SDUV-FEL
}

\author{
FENG Chao ${ }^{1,2}$, ZHANG Meng ${ }^{1}$, LIN GuoQiang ${ }^{1}$, GU Qiang ${ }^{1}$, DENG HaiXiao ${ }^{1}$, CHEN JianHui ${ }^{1}$, \\ WANG Dong ${ }^{1} \&$ ZHAO ZhenTang ${ }^{1 *}$
}

\author{
${ }^{1}$ Shanghai Institute of Applied Physics, Chinese Academy of Sciences, Shanghai 201800, China; \\ ${ }^{2}$ Graduate University of Chinese Academy of Sciences, Beijing 100049, China
}

Received February 28, 2012; accepted April 18, 2012

\begin{abstract}
Cascading stages of high-gain harmonic generation (HGHG) free electron laser (FEL) is a promising way to produce fully coherent X-ray radiation. As a test facility for modern FEL R\&D, the Shanghai deep ultraviolet FEL (SDUV-FEL) is now under upgrading for the cascading two stages of HGHG experiment. Since the energy of the electron beam is as low as about $185 \mathrm{MeV}$ after upgrade, the total harmonic number of this two stages HGHG is only $2 \times 2$, and the wavelength of the final radiation is 196.5 $\mathrm{nm}$ which is the 4th harmonic of the $786 \mathrm{~nm}$ seed laser. With help of three-dimensional simulation codes, design studies on the FEL physics for the cascaded HGHG experiment are present based on the parameters of the upgraded SDUV-FEL facility. It is found from the simulation results that the part of the electron beam which has been used in the first stage can still generate powerful radiation in the radiator of the second stage, and this radiation will be difficult to be separated from the radiation generated by the fresh part of the electron beam. To overcome this problem, a novel method based on the energy spectrum of the electron beam is proposed in this paper to demonstrate the "fresh bunch" technique.
\end{abstract}

cascaded HGHG, FEL experiment, start-to-end simulation, fresh bunch, energy spectrum

Citation: $\quad$ Feng C, Zhang M, Lin G Q, et al. Design study for the cascaded HGHG experiment based on the SDUV-FEL. Chin Sci Bull, 2012, 57: 3423-3429, doi: $10.1007 / \mathrm{s} 11434-012-5379-5$

Free electron lasers (FELs) hold great promise to produce coherent short wavelength radiation with high brightness and ultra-fast time structures which will enable scientists in physics, chemistry, biology and medicine to study nature down to the molecular and atomic level at a time-scale that fits this resolution. Because of its unique performance, FEL is complement to the 3rd generation light source like the Shanghai Synchrotron Radiation Facility (SSRF) [1]. Selfamplified spontaneous emission (SASE) [2,3] and seeded harmonic generation schemes [4-7] are two leading candidates for approaching deep ultraviolet (DUV) to X-ray region. The SASE process produces short wavelength radiation with high peak power and an excellent spatial mode. As the SASE FEL starts from electron beam shot noise, the output radiation typically has poor temporal coherence and

*Corresponding author (email: zhaozt@ sinap.ac.cn) large power fluctuations. An alternative way for generation of fully coherent short wavelength radiation is using the high-gain harmonic generation (HGHG) scheme [4,5]. HGHG consists of two undulators separated by a chicane. The electron beam is first energy modulated by a seed laser in the first short undulator (modulator) and then sent through a chicane (dispersion section) which converts the energy modulation into a density modulation. The density modulated beam is then sent through the second long undulator (radiator) to generate powerful radiation at the high harmonic of the seed frequency. The output property of the HGHG is a direct map of the seed laser's attributes which can have a high degree of temporal coherence and much smaller energy fluctuations than SASE. These theoretical predictions have been demonstrated in the HGHG experiments [8-10]. However, significant bunching at higher harmonics would degrade the quality of the electron beam 
by increasing the beam energy spread in the modulator, which would result in a degradation of the amplification process in the radiator [11]. The need to limit the growth of the energy spread prevents the possibility of reaching short wavelength in a single stage HGHG. For this reason, the cascading stages of HGHG scheme [12] have been proposed. The FEL generated by intermediate radiator can be used as the seed laser for the following stage with the help of the "fresh bunch" technique. Analyses within the framework of idealized models promisingly indicate that cascading several stages of HGHG can produce fully coherent soft X-rays. The cascaded HGHG scheme seems an attractive way for the soft X-ray light source. Several laboratories, including BESSY [13], ELETRRA [9] and SINAP [10,14], proposed the development of deep ultraviolet or soft X-ray FEL based on the cascaded HGHG principle. Among them SDUV has already started installation of a cascaded HGHG FEL system.

The SDUV-FEL is a seeded FEL test facility designed for generating coherent radiation with wavelength down to the ultraviolet region. The design and the relevant R\&Ds of this facility have been under way since 2000 [15], and the two-stage cascading HGHG scheme on the basis of this facility has been proposed several years ago [16]. Recently, the HGHG and EEHG experiments have been successfully carried out [10]. The next goal of this facility is to demonstrate the principle of the cascaded HGHG scheme, including the "fresh bunch" technique. With help of the analytical estimates and the 3D codes, design studies for the cascaded
HGHG scheme based on the upgraded SDUV FEL are present in this paper. It is found in the simulation results that the disturbed part of the electron beam which has been used in the first stage still holds the capability to generate powerful radiation in the beginning of the radiator in the second stage, and it is hard to separate it from the radiation generated by the fresh part of the electron beam when the experimental condition of the second stage has not been optimized. This will be a general problem for the commissioning of all the cascaded HGHG facilities in the future. To overcome this problem, a novel method based on the energy spectrum of the electron beam is proposed to demonstrate the "fresh bunch" technique.

\section{Cascading two stages of HGHG based on SDUV-FEL}

The layout of the upgraded SDUV-FEL is schematically shown in Figure 1. The upgrade is based on the existing facility. The energy of the electron beam has be enhanced from $140 \mathrm{MeV}$ to about $200 \mathrm{MeV}$ when there is no bunch compression, and the operation beam energy will be about $185 \mathrm{MeV}$ when the beam is compressed by a factor of about 2. The linac commissioning has been done after upgrade. Figure 2 shows the measurement results of some main parameters. The energy and energy spread of the electron beam are inferred by measuring the beam central position and beam size on the optical transition radiation (OTR)

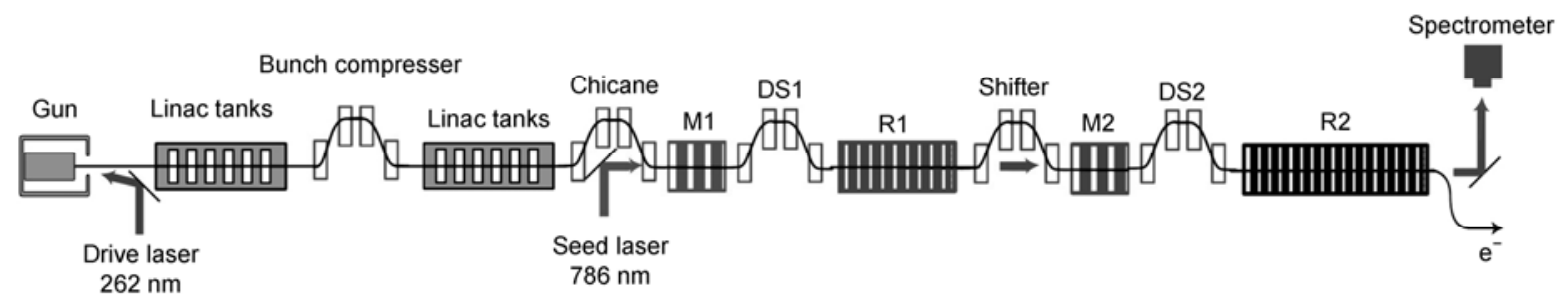

Figure 1 Schematic layout of the upgraded SDUV-FEL. M, modulator; DS, dispersive section; R, radiator.
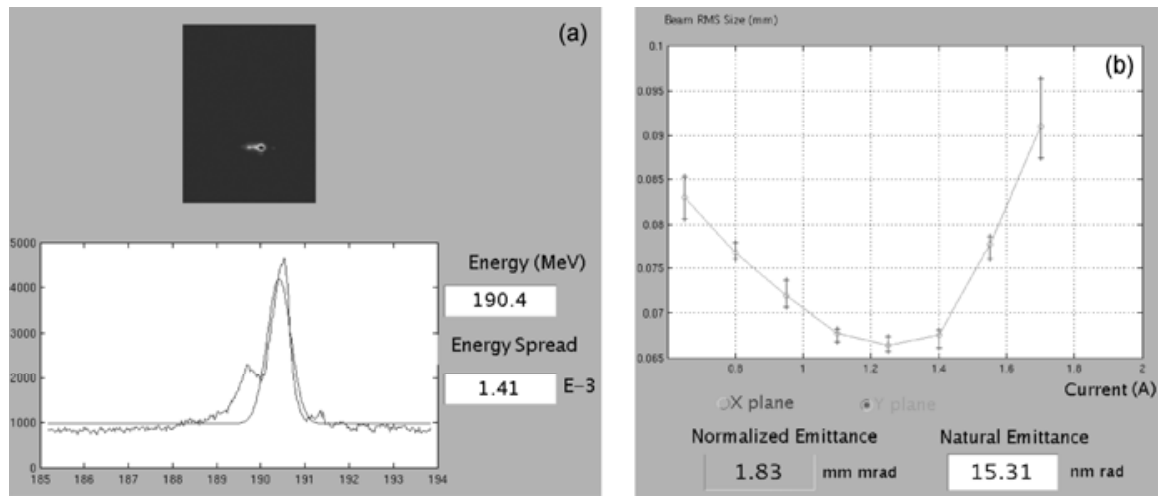

Figure 2 Linac commissioning results. (a) Beam energy and energy spread is measured by the beam energy spectrometer; (b) the normalized emittance is measured with the quadrupole scan technique. 
screen downstream a bending magnet. From Figure 2 (a) it is found that the beam energy is over $190 \mathrm{MeV}$ after compression and the project energy spread is $1.41 \times 10^{-3}$. The normalized emittance of the electron beam is measured with the quadrupole scan technique using the quadrupole triplet and an OTR screen, the normalized emittance is measured to be $1.83 \mathrm{~mm}$ mrad when the bunch charge is about 300 $\mathrm{pC}$.

Besides the linac upgrade, new elements have been added in the undulator system for the cascaded HGHG experiment, including one radiator (R1) in the first stage, one modulator (M2) and one dispersion section (DS2) in the second stage and one shifter between two stages. Main parameters of this upgraded scheme are listed in Table 1 . The first stage $\mathrm{HGHG}$ expects to generate $393 \mathrm{~nm}$ radiation from the $786 \mathrm{~nm}$ seed laser. The FWHM length of the seed laser pulse is about $130 \mathrm{fs}$ which is much shorter than the electron bunch length after compression. So only a small part of the electron beam is modulated in M1 and generates coherent radiation in $\mathrm{R} 1$. This radiation will be shifted to a fresh part of the electron by the shifter and serves as the seed laser for the second stage. A short undulator (M2) with the type of the R1 is employed as the modulator and a small chicane (DS2) is used as the dispersion section in the second stage. Finally, the $196.5 \mathrm{~nm}$ radiation will be generated by the fresh bunch in R2.

\section{Theoretical optimization of cascaded HGHG}

In comparison with the SASE, cascaded HGHG is a multidimensional parameter scheme. The design and optimization of SDUV-FEL cascaded HGHG are achieved using the one dimensional (1D) HGHG theories given by $\mathrm{Yu}$ [4]. According to the HGHG theories, the power of the seed laser and the DS strength are crucial to the HGHG process.

A large seed laser power is necessary for sufficient energy modulation, but at the same time it acts as an additional energy spread that degrades the quality of the electron

Table 1 Machine parameters of the upgraded SDUV-FEL ${ }^{\text {a) }}$

\begin{tabular}{lccc}
\hline & & Stage 1 & Stage 2 \\
\hline \multirow{2}{*}{ Seed laser } & $\tau(\mathrm{FWHM})$ & $130 \mathrm{fs}$ & $\sim 130 \mathrm{fs}$ \\
& $\lambda_{s}(\mathrm{~nm})$ & 786 & 393 \\
\hline \multirow{3}{*}{ Modulator } & $\lambda_{u}(\mathrm{~mm})$ & 50 & 40 \\
& $B_{\text {peak }}(\mathrm{T})$ & $0-1.1$ & $0-0.9$ \\
\hline Dispersion section & $L_{m}(\mathrm{~m})$ & 0.5 & 0.64 \\
\hline \multirow{3}{*}{ Radiator } & $R_{56}(\mathrm{~mm})$ & $0-10$ & $0-10$ \\
& $\lambda_{u}(\mathrm{~mm})$ & 40 & 25 \\
& $B_{\text {peak }}(\mathrm{T})$ & $0-0.9$ & 0.6 \\
& $L_{r}(\mathrm{~m})$ & 4 & 10
\end{tabular}

a) $E=120-200 \mathrm{MeV}, I_{p} \approx 100 \mathrm{~A}, \varepsilon=1.8 \mathrm{~mm} \mathrm{mrad}, \sigma_{\gamma} / \gamma=0.03 \%, 1-2 \mathrm{ps}$ rms length beam. When the total energy spread is too large, the amplification process of the FEL will be saturated. The seed laser of the second stage is produced by the first stage, so how to choose the power of the initial seed laser will be very important. The density modulation of the electron beam can be measured by the bunching factor, which has a maximum value of unity. The bunching factor of $n$th harmonic at the entrance of the gain section can be given by [5]

$$
b_{n}=J_{n}(n D \Delta \gamma) e^{-\frac{1}{2}\left(n D \sigma_{\gamma}\right)^{2}},
$$

where $J_{n}$ is the $n$th order first class Bessel function, $D=$ $k_{s} R_{56} / \gamma$ is a dimensionless parameter related to the dispersive strength of the DS, $k_{s}$ is the wave number of the seed laser, $R_{56}$ is the dispersive strength, $\gamma$ is the reduced beam energy, $\sigma_{\gamma}$ is the local energy spread of the electron beam and $\Delta \gamma$ is the energy modulation amplitude induced by the seed laser which is in proportion to the extraction of the seed laser power $P_{\text {seed }}$.

$$
\begin{aligned}
& \Delta \gamma=k_{s} a_{s} a_{u m} l_{m}[J J]_{1} / \gamma, \\
& a_{s}=\sqrt{\frac{Z_{0} P_{\text {sed }}}{\pi k_{s} Z_{R}\left(m c^{2} / e\right)^{2}}},
\end{aligned}
$$

where $a_{s}$ and $a_{\mathrm{um}}$ are the dimensionless (rms) vector potentials from the seed laser and magnetic field of the modulator, respectively. $l_{m}$ is the length of the modulator, $[J J]_{1}$ is the polarization modification factor for a linearly polarized planar undulator, $Z_{0}=377 \Omega$ is the vacuum impedance and $Z_{R}$ is the Rayleigh length of the seed laser. To get sufficient bunching factor, $n D \sigma_{\gamma}<1$ should be satisfied first; If one wants the nth Bessel function to reach its first and absolute maximum, the requirement is $n D \Delta \gamma \approx n+1$, which means that the energy modulation amplitude should be $n$ times larger than the initial local energy spread. The radiation power can be roughly estimated using the 1D theory of HGHG by

$$
\left\{\begin{array}{l}
P_{\mathrm{coh}}=\frac{\left(Z_{0} a_{u r}[J J]_{1} I b_{n} L_{G}\right)^{2}}{4 \pi \Sigma^{2} \gamma^{2}}, \\
P \approx \frac{3.71}{12} P_{\mathrm{coh}} e^{z / L_{G}} \\
P_{\mathrm{sat}} \approx 1.6 \rho \cdot \frac{\gamma m c^{2} I}{e}
\end{array}\right.
$$

where $P_{\text {coh }}$ is the coherent radiation power in the first two gain lengths of the second undulator, which is proportional to the square of the bunching factor. $a_{u r}$ is the dimensionless parameter of the radiator, $I$ is the beam current, $\Sigma$ is the transverse beam area, $\rho$ is the pierce parameter, $L_{G}=$ $\lambda_{u r} /(4 \sqrt{3} \pi \rho)$ is the gain length in the radiator and $\lambda_{u r}$ is the period length of the radiator. The saturation length in the radiator can be easily deduced by eq. (4): 


$$
L_{\mathrm{sat}} \approx L_{G}\left[\ln \left(\frac{P_{\mathrm{sat}}}{P_{\mathrm{coh}}}\right)+2\right] .
$$

Figure 3 shows the theoretical results of the saturation lengths as a function of the seed laser power for both two stages using eq. (5). It is found that the saturation length of the second stage will not change much when the power of the initial seed laser is larger than $10 \mathrm{MW}$ for our experimental condition. Thus, the power of the seed laser is chosen to be $10 \mathrm{MW}$ for the first stage.

\section{Start-to-end simulation results}

On the basis of the parameters shown in Table 1, start-toend tracking of the electron beam, including all components in Figure 1 has been carried out. The electron beam dynamics in photo-injector was simulated with ASTRA [17] to take into account of space-charge effects. ELEGENT [18] was used for the simulation in the remainder of the linac, while tracking in the undulators was performed with GENESIS [19]. The slice parameters at the exit of the linac are summarized in Figure 4. The beam energy in the central

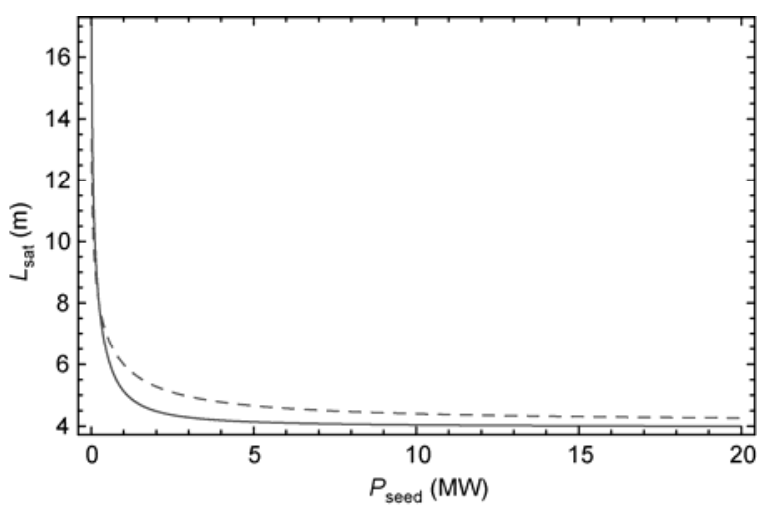

Figure 3 Saturation lengths of the first stage (dashed line) and the second stages (solid line) as a function of the seed laser power in the first stage.
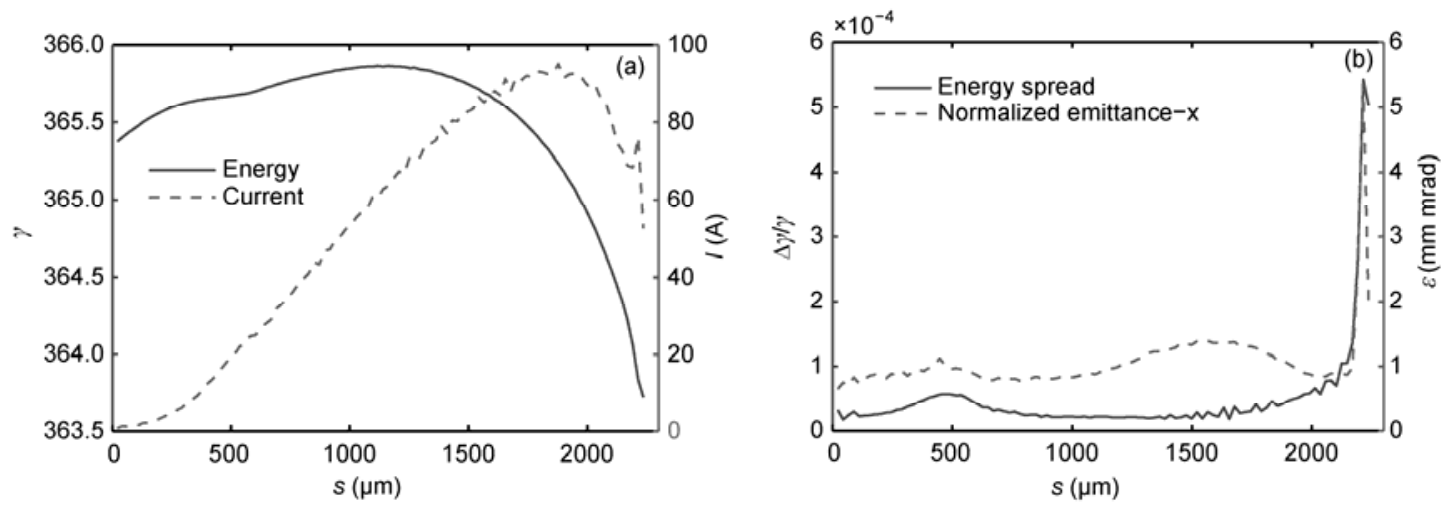

Figure 4 Simulated parameters at the exit of the linac (a) beam energy and current distribution along the electron beam; (b) slice energy spread and slice emittance distribution along the electron beam. part of the electron beam is around $185 \mathrm{MeV}$ and the peak current is about $95 \mathrm{~A}$. A constant profile is maintained in the approximately 1 ps wide and over 80 A region which will be used in the HGHG cascade. The project energy spread of $1.2 \times 10^{-3}$ are predicted from the beam energy curve. A normalized emittance of approximately $1.6 \mathrm{~mm}$ mrad and slice energy spread of about $2.5 \times 10^{-5}$ are observed in Figure 4(b). These simulation results are fit well with the measurement results shown in Figure 2.

The FEL performance of our cascading scheme was simulated by GENESIS based on the output of ELEGENT. A $786 \mathrm{~nm}$ seed pulse with longitudinal Gaussian profile, 10 MW peak power and $130 \mathrm{fs}$ pulse length is adopted as the seed laser of the first stage. To obtain realistic simulation results, the whole electron beam was tracked through the first stage to the second stage HGHG. The simulation results are illustrated in Figure 5. The electron beam is well bunched at the exit of the DS in the first stage. The maximal bunching factor at 2nd harmonic of the seed laser is over 0.4 (Figure 5(a)). The first stage HGHG generates $393 \mathrm{~nm}$ radiation pulse with the output peak power of about $20 \mathrm{MW}$. The length of the seed laser pulse is maintained. For the second stage, the 2nd harmonic bunching factor of the fresh bunch is around 0.4 (Figure 5(b)). The radiation generated by the fresh part saturates after $4 \mathrm{~m}$ with a peak power of 13 MW. One can find in Figure 4(d) that there are two $196 \mathrm{~nm}$ radiation pulses at saturation. The higher one is generated by the fresh part of the electron beam, and the lower one, about $20 \%$ of the higher pulse energy, is generated by the disturbed part of the electron beam which has been strongly bunched in the radiator of the first stage. It is found in eq. (4) that the coherent electron-density modulation, which also has the Fourier components at the harmonics of the radiation generated by the first stage, will still hold the ability for generating powerful coherent harmonic radiation in the first two gain length of the radiator in the second stage. It is necessary to change the strength of the shifter to distinguish the 4th harmonic signal produced by the fresh part from that produced by the disturbed part: the final radiation will be produced only by the disturbed part when the shifter is off, 

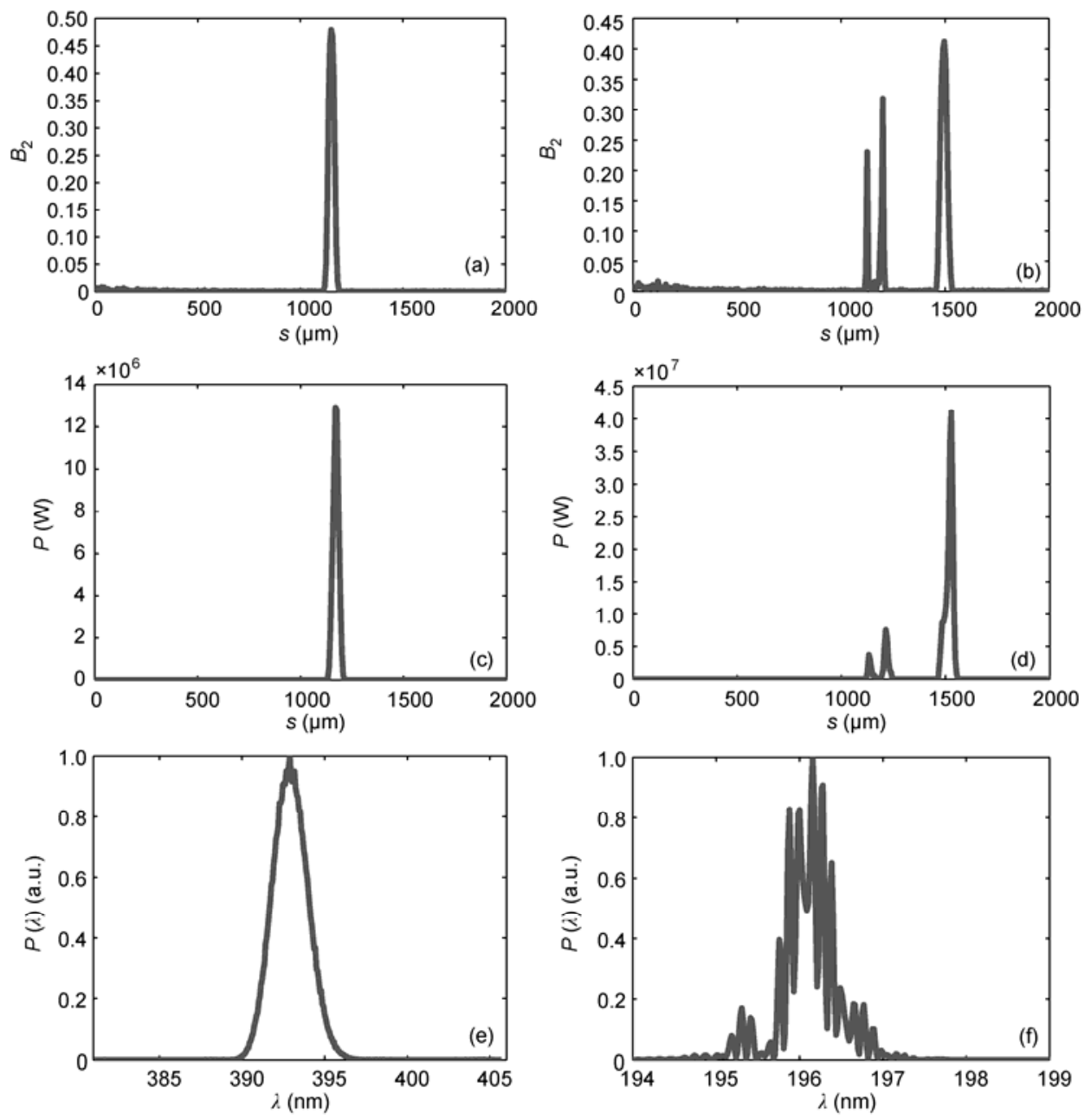

Figure 5 FEL performance in two stages. (a) 2nd harmonic bunching factor distribution along the electron beam at the entrance of the radiator in the first stage; (b) 2nd harmonic bunching factor distribution along the electron beam at the entrance of the radiator in the second stage; (c) output radiation pulse of the first stage; (d) output radiation pulse of the second stage; (e) spectrum of the radiation generated by the first stage; (f) spectrum of the radiation generated by the second stage.

and final radiation will be significantly enhanced when the radiation form the first stage is shifted to a fresh part of the electron beam. The temporal coherence of the final radiation is degraded by the radiation pulse produced by the disturbed part as shown in Figure 4(f).

The energy of the radiation pulse produced by the disturbed part in R2 can be depressed by shifting the disturbed part to a low-current region of the electron beam. This can be achieved by shifting the initial seed laser pulse to the tail part of the electron beam in M1. The radiation power generated by the first stage HGHG will also decrease at the same time. We shifted the seed laser pulse by about $800 \mu \mathrm{m}$ where the beam current is around $30 \mathrm{~A}$. The simulation results are shown in Figure 6. The output power of the first stage decreases to about $3 \mathrm{MW}$, which is still sufficient for the energy modulation in the second stage. It is found in Figure 6(b) that only a single pulse is generated by the second stage. The coherence of the final radiation is also improved.

\section{Demonstration of the "fresh bunch" tech- nique}

Although the radiation generated by the disturbed part of the electron beam in the radiator of the second stage can be depressed by properly choosing the parameters in the simulation, it will be quite difficult to find this condition during the experiment. An alternate way to intuitively demonstrate the principle of the fresh bunch technique is characterization of the energy modulation along the electron bunch. As the part of the electron bunch which has been used for the FEL generation is energy modulated by both the seed laser and the radiation, the energy spread of these parts will be much larger than other parts of the electron beam. Chirping such energy modulated bunch, one would get the peak-to-troughto-peak structures in the energy spectrum, corresponding to variations of the beam energy along the electron bunch.

As Figure 1 shows, there are 4 accelerating tanks and 3 energy spectrometers in our facility. Following "zero- 

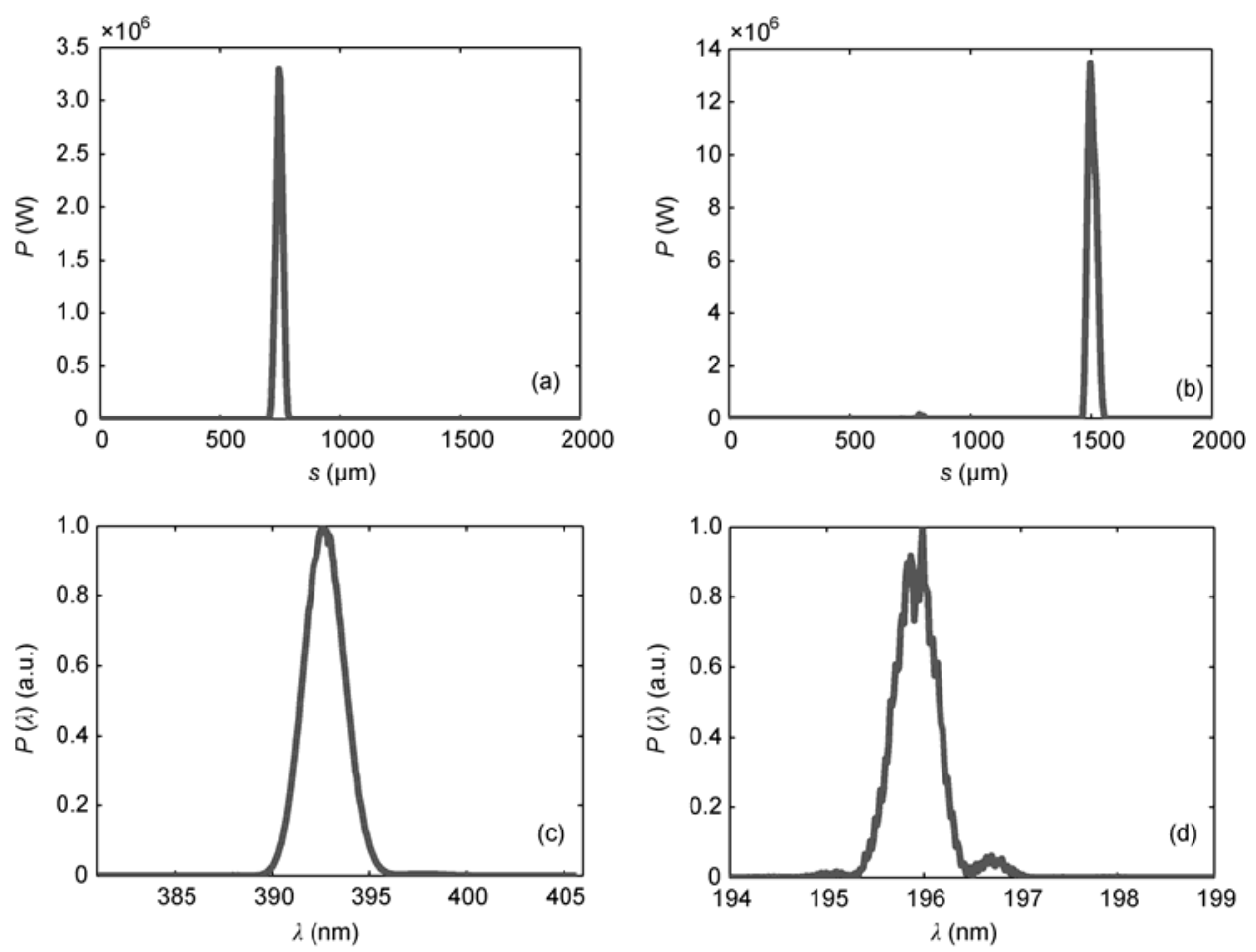

Figure 6 FEL performance when shifting the initial seed laser pulse to the low current part of the electron beam. (a) Output radiation pulse of the first stage; (b) output radiation pulse of the second stage; (c) spectrum of the radiation generated by the first stage; (d) spectrum of the radiation generated by the second stage.

phasing" method, accelerating cavity imparts energy chirp in the bunch, without changing average beam energy. If initially there is no correlation between energy and time, energy spectrum of the chirped bunch (CBE spectrum) exactly represents longitudinal density distribution [20]. The energy spectrum can be scaled in the units of time in the beam rest frame. We adjusted the phase of S4 to imprint considerable positive energy chirp on the electron beam in the simulation. The phase space and energy spectrum of the electron beam at exit of the accelerator is shown in Figure 7. From Figure 7(a), it is found that the energy chirp in the electron beam is nearly linear, and the project energy spread is increased to about $2 \%$. After a horizontal dispersive region, one can get the energy spectrum of the electron beam as Figure 7(b) shows.

Figure 8 shows the longitudinal phase space evolution in the two stages. After the radiator of the first stage, the beam

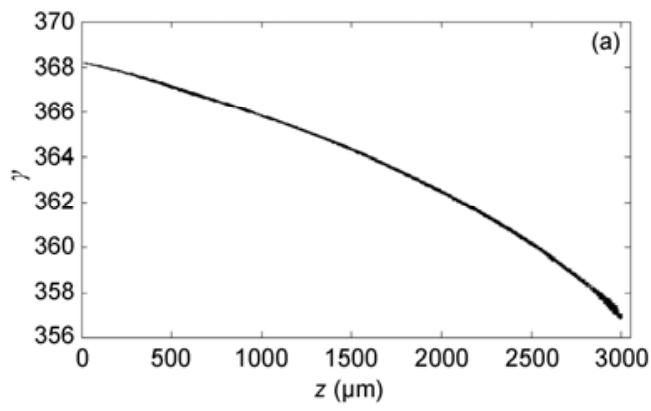

energy of the modulated part is disturbed (Figure 8(a)), the energy of the electrons in this part is changed slightly to a higher or lower value, which will results in a valley and two peaks in the energy spectrum. The electron beam will be separated into two parts on the YAG target after the dipole, as Figure 8(b) shows. After passing through the radiator of the second stage, the fresh part is also disturbed by interacting with the powerful radiation (Figure $8(\mathrm{c})$ ), and the electron will be separated into three parts (Figure 8(d)), which clearly demonstrates the fresh bunch technique.

\section{Conclusions}

We present design studies of cascaded HGHG experiment based on the upgraded SDUV-FEL. The results show that the proof-of-principle experiments of cascaded HGHG can

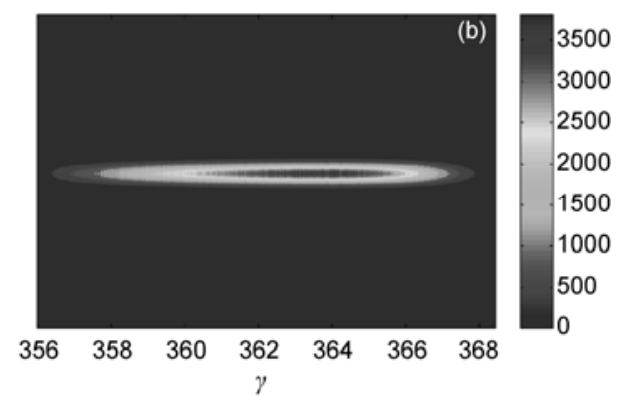

Figure 7 Phase space (a) and energy spectrum (b) of the electron beam at the exit of the accelerator. 

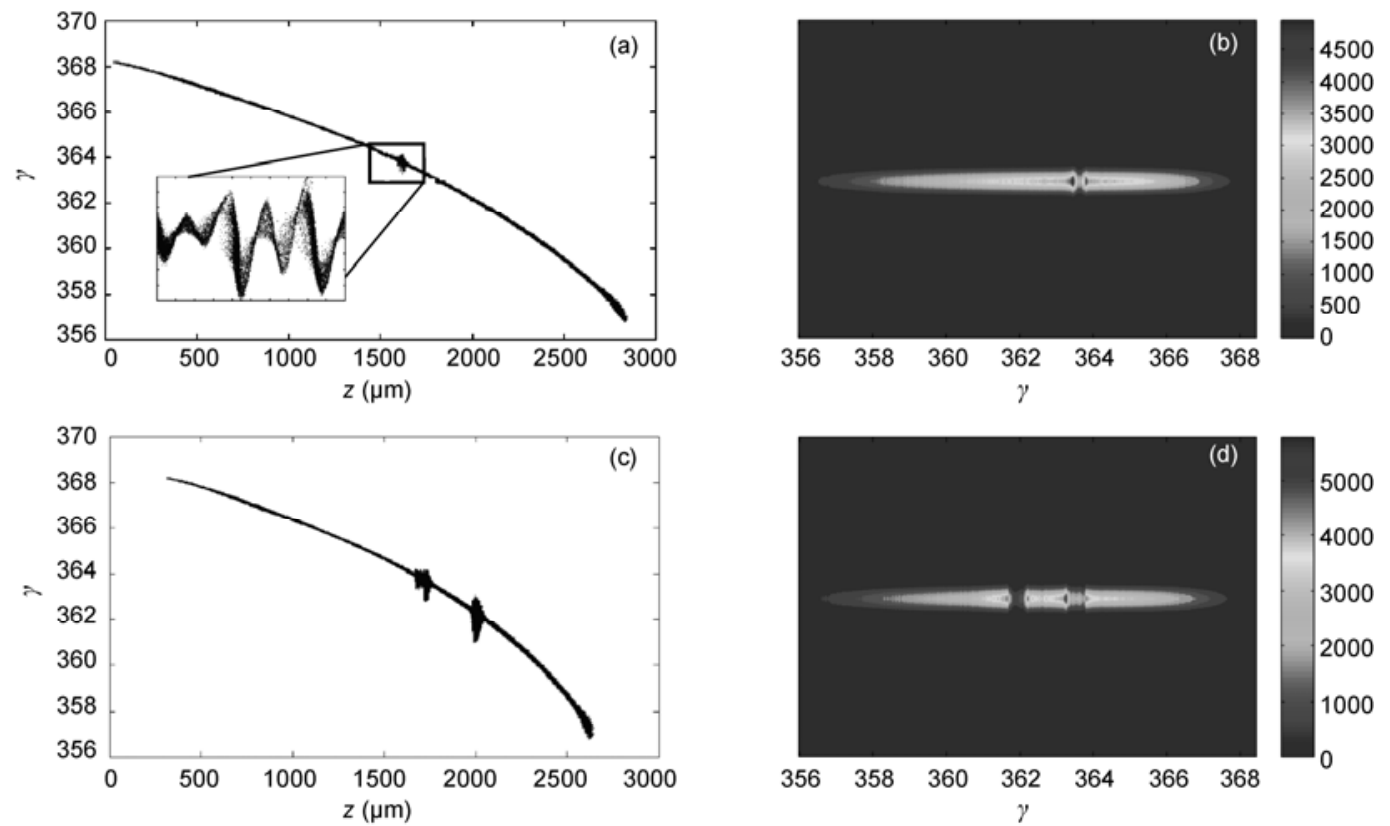

Figure 8 Phase space (a) and energy spectrum (b) of the electron beam at the exit of the first stage; phase space (c) and energy spectrum (d) of the electron beam at the exit of the second stage.

be carried out at our facility with an additional stage of HGHG. The radiation of the cascaded HGHG at $196.5 \mathrm{~nm}$ will reach saturation in the second stage. From the simulation results, it is also found that the micro-bunching generated by the first stage will still have the capability to release powerful radiation in the beginning of the radiator in the second stage, and it is hard to separate it from the radiation generated by the fresh part of the electron beam. To overcome this problem, a novel method based on the energy spectrum of the electron beam is proposed to demonstrate the fresh bunch technique. Simulation results indicate that this method have a high resolution and will be easy to be carried out.

The authors would like to thank L.H. Yu (BNL) for helpful discussion and comments. This work was supported by the National Natural Science Foundation of China (10935011) and the National Basic Research Program of China (2011CB808300).

1 Jiang M H, Yang X, Xu H J, et al. Shanghai Synchrotron Radiation Facility. Chin Sci Bull, 2009, 54: 4171-4181

2 Kondratenko A M, Saldin E L. Generation of coherent radiation by a relativistic electron beam in an undulator. Part Accel, 1980, 10: 207-216

3 Bonifacio R, Pellegrini C, Narducci L M. Collective instabilities and high-gain regime in a free electron laser. Opt Commun, 1984, 50: 373-378

4 Yu L H. Generation of intense UV radiation by sub-harmonically seeded single-pass free-electron lasers. Phys Rev A, 1991, 44: 5178-5193

5 Yu L H, Ben-Zvi I. High-gain harmonic generation of soft X-rays with the "fresh bunch" technique. Nucl Instr Meth A, 1997, 393: 96-99

6 Stupakov G. Using the beam-echo effect for generation of shortwavelength radiation. Phys Rev Lett, 2009, 91: 074801
7 Feng C, Zhao Z T. Hard X-ray free-electron laser based on echoenabled staged harmonic generation scheme. Chin Sci Bull, 2010, 55: 221-227

8 Yu L H, DiMauro L, Doyuran A, et al. First ultraviolet high-gain harmonic-generation free-electron laser. Phys Rev Lett, 2003, 102: 074801

9 Allaria E. FEL commissioning at FERMI@Elettra. In: Proceedings of FEL 2011, Shanghai, China, WEOBI1

10 Zhao Z T. Seeding experiments at the SDUV-FEL. In: Proceedings of FEL 2011, Shanghai, China, WEOBI2

11 Brefeld W, Faatz B, Feldhaus J, et al. Study of the frequency multiplication process in a multistage HGHG FEL. Nucl Instr Meth A, 2002, 483: 80-88

$12 \mathrm{Wu} \mathrm{J} \mathrm{H}, \mathrm{Yu} \mathrm{L} \mathrm{H}$. Coherent hard X-ray production by cascading stages of high gain harmonic generation. Nucl Instr Meth A, 2001, 475: 104-111

13 Abo-Bakr M, Anders W, Bahrdt J, et al. STARS-An FEL to demonstrate cascaded HGHG. In: Proceedings of FEL 2007, Novosobirsk, Russia, TUBAU03

14 Zhao Z T, Chen S Y, Yu L H, et al. Shanghai soft X-ray free electron laser test facility. In Proceedings of IPAC2011, San Sebastian, Spain, THPC053

15 Zhao Z T, Dai Z M, Zhao X F, et al. The Shanghai high-gain harmonic generation DUV free-electron laser. Nucl Instr Meth A, 2004, 528: 591-594

16 Deng H X, Dai Z M. Design of cascading two stages of high gain harmonic generation scheme based on Shanghai deep ultraviolet free electron laser. Chin Phys C, 2008, 32: 236-242

17 Floettmann K. ASTRA user's manual, 1999, http://www.desy.de/ mpyflo/Astra_dokumentation

18 Borland M. Elegant: A flexible SDDS-compliant code for accelerator simulation. Advanced Photon Source LS-287, 2000

19 Reiche S. GENESIS 1.3: A fully 3D time-dependent FEL simulation code. Nucl Instr Meth A, 1999, 429: 243-248

20 Shaftan T, Yu L H. Analysis of space charge driven modulation in electron bunch energy spectra. BNL Preprint, 2003, BNL-714902003-CP

Open Access This article is distributed under the terms of the Creative Commons Attribution License which permits any use, distribution, and reproduction in any medium, provided the original author(s) and source are credited. 